\title{
Molecular Modeling and Reactivity of Thermally Altered Coals by Molecular Simulation Techniques
}

Yungang Zhao, ${ }^{\dagger}$ Shaoqing Wang,,${ }^{\dagger}{ }^{\dagger}$ Yu Liu,${ }^{\dagger}$ Xiaoxia Song,,${ }^{\ddagger}$ Hao Chen,${ }^{\dagger}$ Xiaomei Zhang,,${ }^{\dagger}$ YuHan Lin, ${ }^{\dagger}$ and Xiaoling Wang ${ }^{\dagger}$

${ }^{\dagger}$ College of Geoscience and Surveying Engineering, China University of Mining and Technology (Beijing), Beijing 100083, China

${ }^{\ddagger}$ College of Mining Engineering, Taiyuan University of Technology, Taiyuan 030024, China

\section{Supporting information}

Table S1. ${ }^{13} \mathrm{C}$ NMR peak-fitting results of SL-1

\begin{tabular}{cccc}
\hline Peak number & Chemical shift/ppm & Molar content/\% & Assignment \\
\hline 1 & 6.57 & 0.95 & Aliphatic methyl \\
2 & 15.22 & 1.35 & Aliphatic methyl \\
3 & 20.76 & 0.64 & Aromatic methyl \\
4 & 30.64 & 1.23 & Methylene \\
5 & 42.53 & 0.36 & Methine \\
6 & 47.04 & 0.12 & Quaternary carbons \\
7 & 82.32 & 0.78 & Oxy-quaternary \\
8 & 94.93 & 0.31 & Anomeric carbon \\
9 & 107.84 & 2.91 & Ortho-oxyaromatic protonated \\
10 & 116.27 & 9.12 & Ortho-oxyaromatic branched \\
11 & 122.16 & 22.09 & Protonated aromatics \\
12 & 127.80 & 27.70 & Protonated aromatics \\
13 & 132.54 & 16.26 & Bridgehead aromatics \\
14 & 139.73 & 9.68 & Alkyl-substituted aromatics
\end{tabular}




\begin{tabular}{cccc}
15 & 148.22 & 2.81 & Oxygen-substituted aromatics \\
16 & 160.66 & 0.55 & Oxygen-substituted aromatics \\
17 & 168.20 & 0.13 & Carbonyl in carboxyl, ester and amide \\
18 & 180.33 & 0.37 & Carbonyl in carboxyl, ester and amide \\
19 & 188.00 & 0.31 & Carbonyl in ketone, aldehyde and quinone \\
20 & 205.87 & 0.66 & Carbonyl in ketone, aldehyde and quinone \\
21 & 215.99 & 1.67 & Carbonyl in ketone, aldehyde and quinone \\
\hline
\end{tabular}

Table S2. ${ }^{13} \mathrm{C}$ NMR peak-fitting results of SL-2

\begin{tabular}{cccc}
\hline Peak number & Chemical shift/ppm & Molar content $/ \%$ & Assignment \\
\hline 1 & 12.62 & 0.80 & Aliphatic methyl \\
2 & 18.46 & 0.32 & Aromatic methyl \\
3 & 28.43 & 0.41 & Methylene \\
4 & 33.27 & 0.36 & Methylene \\
5 & 42.38 & 0.20 & Methine \\
6 & 46.87 & 0.24 & Quaternary carbons \\
7 & 64.34 & 0.86 & Oxy-methylene \\
8 & 72.80 & 0.31 & Oxy-methine \\
9 & 80.25 & 0.78 & Oxy-quaternary \\
10 & 96.43 & 3.46 & Anomeric carbon \\
11 & 110.00 & 4.77 & Ortho-oxyaromatic protonated \\
12 & 117.11 & 10.45 & Ortho-oxyaromatic branched \\
13 & 122.09 & 21.30 & Protonated aromatics \\
14 & 127.79 & 24.92 & Protonated aromatics \\
15 & 131.65 & 14.42 & Bridgehead aromatics \\
16 & 138.95 & 9.83 & Alkyl-substituted aromatics \\
17 & 147.64 & 4.51 & Oxygen-substituted aromatics \\
18 & 158.77 & 0.68 & Oxygen-substituted aromatics \\
19 & 164.68 & 0.35 & Carbonyl in carboxyl, ester and amide \\
20 & 170.64 & 0.45 & Carbonyl in carboxyl, ester and amide \\
21 & 177.89 & 0.11 & Carbonyl in carboxyl, ester and amide \\
22 & 184.06 & 0.28 & Carbonyl in ketone, aldehyde and quinone \\
23 & 196.57 & 0.19 & Carbonyl in ketone, aldehyde and quinone \\
\hline
\end{tabular}

Table S3. ${ }^{13} \mathrm{C}$ NMR peak-fitting results of SL-3

\begin{tabular}{cccc}
\hline Peak number & Chemical shift $/ \mathrm{ppm}$ & Molar content $/ \%$ & Assignment \\
\hline 1 & 0.37 & 0.30 & Aliphatic methyl \\
2 & 5.56 & 0.43 & Aliphatic methyl
\end{tabular}




\begin{tabular}{cccc}
3 & 12.29 & 0.39 & Aliphatic methyl \\
4 & 60.24 & 0.61 & Oxy-methyl \\
5 & 68.78 & 0.40 & Oxy-methylene \\
6 & 76.57 & 0.98 & Oxy-methine \\
7 & 88.07 & 1.18 & Oxy-quaternary \\
8 & 95.53 & 1.04 & Anomeric carbon \\
9 & 104.45 & 4.09 & Ortho-oxyaromatic protonated \\
10 & 115.35 & 13.25 & Ortho-oxyaromatic branched \\
11 & 121.66 & 22.48 & Protonated aromatics \\
12 & 127.53 & 25.29 & Protonated aromatics \\
13 & 132.14 & 15.37 & Bridgehead aromatics \\
14 & 139.79 & 9.01 & Alkyl-substituted aromatics \\
15 & 150.36 & 3.12 & Oxygen-substituted aromatics \\
16 & 162.65 & 0.45 & Oxygen-substituted aromatics \\
17 & 169.97 & 0.77 & Carbonyl in carboxyl, ester and amide \\
18 & 179.08 & 0.29 & Carbonyl in carboxyl, ester and amide \\
19 & 187.91 & 0.39 & Carbonyl in ketone, aldehyde and quinone \\
20 & 194.73 & 0.15 & Carbonyl in ketone, aldehyde and quinone \\
\hline
\end{tabular}

